\title{
The Microbiome and Diet: Friend or Foe in the Future Treatment of Inflammatory Bowel Disease?
}

Udai P Singh*

Pathology, Microbiology and Immunology, School of Medicine, University of South Carolina, Columbia, SC 29208, USA

"Corresponding author: Udai P Singh, Pathology, Microbiology and Immunology, School of Medicine, University of South Carolina, Columbia, SC 29208, USA, Tel: +1-803-216-3423; Fax: +1-803-216-3413; E-mail: udai.singh@uscmed.sc.edu

Received date: August 22, 2015; Accepted date: August 24, 2015; Published date: September 01, 2015

Copyright: @ 2015 Singh UP. This is an open-access article distributed under the terms of the Creative Commons Attribution License, which permits unrestricted use, distribution, and reproduction in any medium, provided the original author and source are credited.

\begin{abstract}
It is well established that uncontrolled activation of effector immune cells in response to the gut microbiota triggers chronic inflammatory bowel disease (IBD) symptoms in genetically susceptible hosts. The exact cause(s) for IBD remains unknown. Previous studies have examined, in detail, how the gut microbiome, genetic susceptibility, and environmental stress may activate the immune responses associated with IBD. Recent studies show that a high fat diet alters the gut microbiota structure and function and facilitates the initiation and progression of immune responses responsible for IBD outcome. Some recent studies also clearly suggest that changes in pathobionts lead to an altered immune response and disease progression. This editorial focuses on some information provided in these studies and addresses how high fat diet induced alteration in the gut microbiota affect IBD progression. We provide some suggestions on how our current knowledge might be used to develop new dietary microbial and immune based modulation strategies for risk assessment and treatment of IBD.
\end{abstract}

\section{Description}

The intestine harbors nearly 100 trillion commensal bacteria that exert crucial effects on overall human health. Genetic studies on the community of gut microbes have shown that they have a role in the development of inflammatory bowel disease (IBD). They have also provided important landmarks in studies of IBD based on recent technological advances in sequencing, genomics, and bioinformatics. The microbiome is a key factor in establishing a balance between immune responses and tolerance to inflammation in the intestinal environment; that balance is required to maintain gut homeostasis.

Since the nineteenth and twentieth centuries, it has been recognized that the intestinal flora, including bacterial pathogens, are involved in the pathogenesis of IBD [1]. This conclusion is supported by several models of IBD in which animals did not develop symptoms of colitis in a germ-free environment. Further, almost 100 years ago, on the basis of clinical and pathologic similarities [2], an association was suggested among the microbiome, Crohn's disease (CD), which is a form of IBD, and chronic inflammatory enteropathy in ruminants and other animals. The main symptoms of IBD in humans and animal models contain granulomas and, at that time, Mycobacterium avium subsp. paratuberculosis (MAP) was thought to act as an infectious etiologic agent to induce chronic inflammation [3]. This was also associated with the observation that MAP was identified in milk from infected cows [4], as well as modern-day water supplies [5]. The isolation of MAP from intestinal biopsies of patient with CD [6,7] also suggests that MAP plays a role in $\mathrm{CD}$. We have shown a plausible mechanism of Ag-specific T-cell responses in experimental colitis driven by potent Ags conserved in MAP species [8]. This finding, together with recent identification and characterization of various microbiome species in the colon and ileum that clearly induce IBD or decrease its severity in humans opens a new paradigm for this field.

Over the past half-century, the incidence of IBD has been increasing in Western society at an alarming rate. Dietary intake, smoking, alcohol consumption, and altered sleep habits are the main triggers of IBD [9]. Dietary intake mediates microbiome species that affect diverse regulatory mechanisms, including both innate and adaptive immunity during the development of IBD [10]. Accumulating evidence suggests that the immunological lesions of IBD are accompanied by prominent infiltrates of cells, including $\mathrm{T}$ lymphocytes [11], macrophages, and neutrophils [12], mediated in part by changes in the gut microbiota. The chronic inflammation associated with IBD can be initiated by the emergence of proinflammatory pathobionts and a decline in the number of commensal microbiota $[13,14]$.

Our interest in complementary and alternative medicine, together with a recent report that resveratrol, a plant-derived compound, improves gut microbiota dysbiosis induced by a high fat diet [15] as compared to regular diet, brings our attention to assessment of dietinduced dysbiosis as a driver of IBD pathogenesis. Changes in modern human dietary patterns, especially the widespread consumption of a diet high in fats and sugar, has changed the microbial community of the gut.

This is supported by a study showing that children with high-fat or plant-based diets have vastly different gut microbes than do those on a rich sugar and fat diet [16]. Another study has provided evidence that children who had received antibiotics had a greater risk of IBD than did those who had not, supporting observations that the microbial community affects the development and progression of IBD [17]. Similarly, in experimental model of spontaneous colitis, feeding subjects a Western diet rich in saturated milk fat worsened disease symptoms through an increase in the number of Th1 cells [18]. On the other hand, humans in a clinical study who were placed on either an meat-based diet and/or a plant-based diet showed increases in biletolerant microbes and decreases in firmicutes, which metabolize plant polysaccharides [19]. Further, a diet based on animal products reduced short-chain fatty acids (SCFAs) as a result of having a low dietary 
content of plant fibers [19]. This decrease in microbial production of SCFAs, which correlates with a decrease in mucosal barrier function, as well as variation in regulatory $\mathrm{T}$-cell function (Tregs), increases disease severity [20]. Similarly, bacterial strains of Clostridia induce clustering of induced colonic Tregs that reduce the severity of experimental colitis [21]. This may be mediated by increased bioavailability of SCFAs, which induce TGF- $\beta$ to mediate Tregs. These findings and other evidence clearly suggest that increased intake of high-fat Western diets is a critical factor in facilitating changes in the gut microbiome that then trigger autoimmune diseases such as IBD in humans.

Recent advances in understanding immune and microbiome interactions add an exciting new perspective to our understanding on the development of IBD and provide an avenue for possible new treatments. Further, based on the current extent of our knowledge of how diet alters immune response and tolerance across the population, it continues to be very difficult to translate results into conclusions and treatments. Thus, dietary modulation and its impact on the gut microbiome should be examined in detail in many experimental models of intestinal inflammation. This essential if we are to reach prudent, practical conclusions. However, the next major task for clinicians is to advance current understanding of host-microbe interactions that modify the gut microbiota and that may be important in the context of human diet.

\section{References}

1. Krasner WL (1988) New directions in Medicare and Medicaid managed care. Med Staff Couns 2: 23-29.

2. Chiodini RJ (1989) Crohn's disease and the mycobacterioses: a review and comparison of two disease entities. Clin Microbiol Rev 2: 90-117.

3. Harris NB, Barletta RG (2001) Mycobacterium avium subsp. paratuberculosis in Veterinary Medicine. Clin Microbiol Rev 14: 489-512.

4. Lombard JE, Garry FB, McCluskey BJ, Wagner BA (2005) Risk of removal and effects on milk production associated with paratuberculosis status in dairy cows. J Am Vet Med Assoc 227: 1975-1981.

5. Pickup RW, Rhodes G, Arnott S, Sidi-Boumedine K, Bull TJ, et al. (2005) Mycobacterium avium subsp. paratuberculosis in the catchment area and water of the River Taff in South Wales, United Kingdom, and its potential relationship to clustering of Crohn's disease cases in the city of Cardiff. Appl Environ Microbiol 71: 2130-2139.

6. Autschbach F, Eisold S, Hinz U, Zinser S, Linnebacher M, et al. (2005) High prevalence of Mycobacterium avium subspecies paratuberculosis IS900 DNA in gut tissues from individuals with Crohn's disease. Gut 54: 944-949.
7. Naser SA, Ghobrial G, Romero C, Valentine JF (2004) Culture of Mycobacterium avium subspecies paratuberculosis from the blood of patients with Crohn's disease. Lancet 364: 1039-1044.

8. Singh UP, Singh S, Singh R, Karls RK, Quinn FD, et al. (2007) Influence of Mycobacterium avium subsp. paratuberculosis on colitis development and specific immune responses during disease. Infection and immunity 75: 3722-3728.

9. Montgomery SM, Ekbom A (2002) Epidemiology of inflammatory bowel disease. Curr Opin Gastroenterol 18: 416-420.

10. Turnbaugh PJ, Bäckhed F, Fulton L, Gordon JI (2008) Diet-induced obesity is linked to marked but reversible alterations in the mouse distal gut microbiome. Cell Host Microbe 3: 213-223.

11. Powrie F, Leach MW (1995) Genetic and spontaneous models of inflammatory bowel disease in rodents: evidence for abnormalities in mucosal immune regulation. Ther Immunol 2: 115-123.

12. Selby WS, Janossy G, Bofill M, Jewell DP (1984) Intestinal lymphocyte subpopulations in inflammatory bowel disease: an analysis by immunohistological and cell isolation techniques. Gut 25: 32-40.

13. Manichanh C, Rigottier-Gois L, Bonnaud E, Gloux K, Pelletier E, et al. (2006) Reduced diversity of faecal microbiota in Crohn's disease revealed by a metagenomic approach. Gut 55: 205-211.

14. Round JL, Mazmanian SK (2009) The gut microbiota shapes intestinal immune responses during health and disease. Nat Rev Immunol 9: 313-323.

15. Qiao Y, Sun J, Xia S, Tang X, Shi Y, et al. (2014) Effects of resveratrol on gut microbiota and fat storage in a mouse model with high-fat-induced obesity. Food Funct 5: 1241-1249.

16. De Filippo C, Cavalieri D, Di Paola M, Ramazzotti M, Poullet JB, et al. (2010) Impact of diet in shaping gut microbiota revealed by a comparative study in children from Europe and rural Africa. Proc Natl Acad Sci U S A 107: 14691-14696.

17. Hviid A, Svanström H, Frisch M (2011) Antibiotic use and inflammatory bowel diseases in childhood. Gut 60: 49-54.

18. Devkota S, Wang Y, Musch MW, Leone V, Fehlner-Peach H, et al. (2012) Dietary-fat-induced taurocholic acid promotes pathobiont expansion and colitis in Il10-/- mice. Nature 487: 104-108.

19. David LA, Maurice CF, Carmody RN, Gootenberg DB, Button JE, et al. (2014) Diet rapidly and reproducibly alters the human gut microbiome. Nature 505: 559-563.

20. Smith PM, Howitt MR, Panikov N, Michaud M, Gallini CA, et al. (2013) The microbial metabolites, short-chain fatty acids, regulate colonic Treg cell homeostasis. Science 341: 569-573.

21. Atarashi K, Tanoue T, Oshima K, Suda W, Nagano Y, et al. (2013) Treg induction by a rationally selected mixture of Clostridia strains from the human microbiota. Nature 500: 232-236. 